\title{
STUDIES OF THE COHERENT HALF-INTEGER RESONANCE
}

\author{
S. Cousineau, Indiana University, Bloomington, IN, USA \\ J. Holmes, J. Galambos, Oak Ridge National Laboratory, Oak Ridge, TN, USA. \\ R. Macek, Los Alamos National Laboratory, Los Alamos, NM, USA \\ A. Fedotov, J. Wei, Brookhaven National Laboratory Upton, NY, USA
}

\begin{abstract}
We present studies of space-charge-induced beam profile broadening at high intensities in the Proton Storage Ring (PSR) at Los Alamos National Laboratory. Previous work has associated the observed broadening in the vertical direction with the coherent half integer resonance [1]. Here, we study the effect of the space charge environment on this resonance; specifically, we investigate the strength of the resonance versus beam intensity, longitudinal bunching factor, transverse lattice tune, and two different beam injection scenarios. For each case, detailed particle-in-cell simulations are combined with experimental results to elucidate the behavior and sensitivity of the beam resonance response.
\end{abstract}

\section{INTRODUCTION}

The next generation of high intensity synchrotrons will require unprecedented minimization of beam loss in order to control radiation activation of the machine. Many of these accelerators will operate in an energy regime where space charge effects are a primary mechanism for halo development and beam loss. In striving towards successively higher beam intensities, an important task is to understand space charge from both theoretical and experimental standpoints. The Proton Storage Ring (PSR) at Los Alamos National Laboratory can operate with very high space charge effects and therefore provides an ideal setting for this type study. In this paper, we analyze experimentally observed beam broadening at high intensities in the PSR ring. Both particle core model and particle-in-cell (PIC) simulations are employed as tools to interpret the data. In the PIC simulations, we pay particular attention to lattice, injection, and RF settings, and in general find very good agreement between the simulated and experimentally measured beam profiles.

The quantity of merit in space charge studies has traditionally been the incoherent space charge tune shift limit. Recently, experimental and computational work has shown that the coherent tune bears more consequence on resonance behavior of the beam [2,3]. A complete analysis of the half-integer coherent resonance response was performed by Sacherer in his doctoral thesis [4], and we quote his results extensively here. He used the particle core model to demonstrate that the onset of the half-integer resonance occurs at space charge tune shifts in excess of the incoherent tune limit. He later extended his analysis to rms second moments of the beam distribution, thus making it applicable to realistic space charge scenarios. We have performed extensive work to tie Sacherer's core model together with an independently developed rms core model which includes dispersion $[5,6]$ and also with PIC calculations and experimental data. The emphasis here is weighted heavily towards the experimental side.

\section{BEAM BROADENING AT $\mathbb{H I G H}$ INTENSITY AT PSR}

The PSR ring is 90 meters in length an can accumulate up to about $5 \times 10^{13}$ protons. Beam profile broadening is observed at the highest operating intensities (above $3 \times 10^{13}$ ) and coincides with an escalation of beam losses. For the experiment presented here, $7 \mu \mathrm{C}$ of beam was accumulated over a period of $1.16 \mathrm{~ms}$, or about 3214 turns, injecting $1.36 \times 10^{10}$ protons per turn. The lattice tunes were set to their nominal working values of $\left(\nu_{x}, \nu_{y}\right)=$ $(3.19,2.19)$, and optimal injection settings with vertical painting were used. Horizontal and vertical profiles were measured on a wire scanner in the extraction channel after the full accumulation of beam. The intensity of the beam was varied by chopping the linac beam pulses before injection into the ring. The profiles in Figure 1 show the vertical beam profile at one-fourth of the full intensity, one-half of the full intensity, and at the full intensity. No significant difference is observable between the one-fourth and one half intensity profiles, whereas the full intensity case exhibits a large amount of beam broadening.

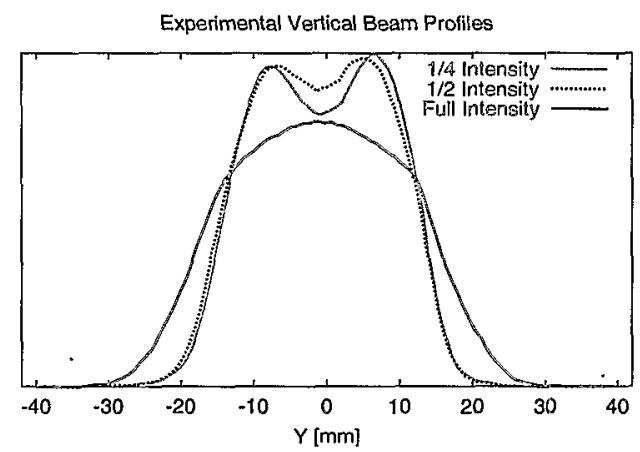

Figure 1: Vertical beam profiles measured on a wire scanner after extraction of the accumulated beam, shown for one-fourth, one-half, and full intensity beams.

In order to explore the area between the half and the full intensities, we invoke PIC model simulations of the full ex- 
periment. The simulations neglect magnetic errors, strip foil scattering, and chromatic effects, but include a complete set of transverse and longitudinal space charge interactions; therefore, space charge provides the only nonlinearity. The details of the code are available in the ORBIT reference manual [7]. Although the benchmarks are not shown here, good agreement is reached for each of the profiles shown in Figure 1, as well as for the other experimental profiles discussed below.

Two quantities that are of importance in diagnosing space-charge-induced effects on a beam are the spacecharge-depressed incoherent tunes, and the second moment of the beam, both available to us through the PIC simulations. Figure 2 below shows the former, plotted as a function of longitudinal distance in the ring, for both the half intensity and full intensity cases. The PSR longitudinal profile is sharply peaked in the center, leading to large tune depressions in this area. For the half size beam, the maximally depressed tune shifts slightly exceed the incoherent half-integer threshold, $\nu_{y}=2.0$. Recall that no beam broadening occurs at this stage, implying that the incoherent threshold can be crossed without immediate consequence to the beam. At the full intensity, where the broadening is seen, the beam has crossed the incoherent limit by an additional 0.1. The limit for the onset of beam broadening relative to the tune shift points strongly towards a coherent response to the resonance condition.

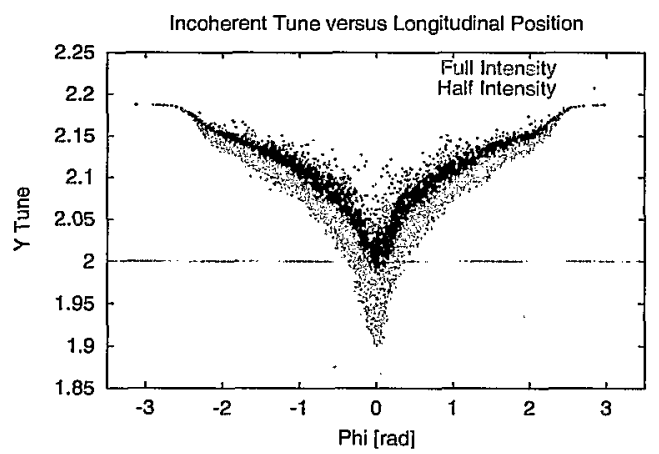

Figure 2: Incoherent tune shifts plotted as a function of longitudinal coordinate in the ring for full and half intensity beams. The dashed line corresponds to the half-integer stopband.

Further evidence of the coherent resonance is presented in Figure 3, where the second order moments of the beam, normalized to the matched beam envelope, are plotted over one turn of the accelerator. Here, the rms beam envelope executes four oscillations per turn, a signature trait of the half-integer coherent resonance. The moments are plotted for particles in the middle of the longitudinal distribution, where the density is highest. An analysis of the moments outside of this range shows a sharp decrease in the moment amplitudes, and frequencies tending towards twice the bare tunes as we move away from the center. This is an indication that the observed beam profile broadening re- sults mainly from particles located at the very center of the longitudinal bunch, a fact which makes for interesting experiments related to the longitudinal bunching factor.

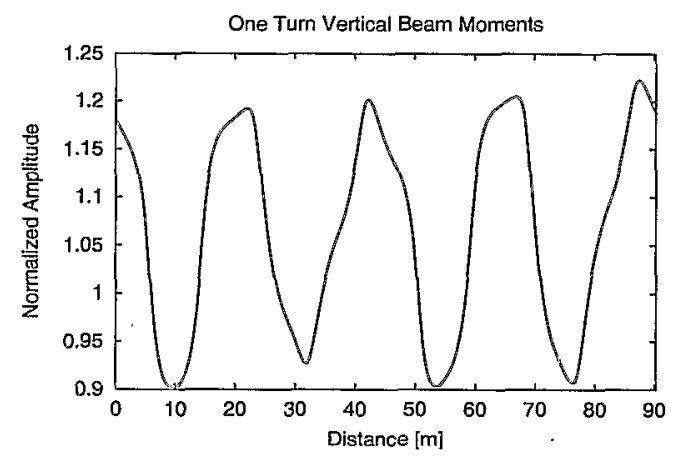

Figure 3: Normalized second moments of the full intensity vertical beam, plotted over one turn of the ring.

\section{EXPLORATIVE EXPERIMENTS}

This section is dedicated to three experiments performed to explore the onset of the resonance in detail. In the first experiment, the longitudinal profile shape was altered by placing a notch in the center of the longitudinal profile, i.e., no beam was injected within 15 degrees of the center of the bunch train; additional beam was injected outside of this range so that the final intensity of the beam was unaffected. Beam eventually diffused into the notched region, but the overall peaking was greatly reduced, and the bunching factor increased from the nominal value of about 0.3 to about 0.45 . The effect on the transverse profiles is shown in Figure 4 , where the vertical profiles after the full accumulation with and without the longitudinal notch are plotted. Note that the longitudinally notched beam is about $15 \%$ thinner. Full PIC simulations of the experiment show that the emittance growth for the highest intensity case is greatly reduced when the notch is in place, with the final rms emittance reaching about $7 \pi \mathrm{mm} \cdot \mathrm{mrad}$ with the notch, compared to the $9.5 \pi \mathrm{mm} \cdot \mathrm{mrad}$ reached without the notch. The notch was not observed to make any difference in the profiles in lower intensity cases, where the beam is not near the resonance.

In the second experiment, the horizontal tune was kept at the fixed value of $\nu_{x}=3.19$, while the vertical tune was incrementally lowered from the nominal $\nu_{y}=2.19$ to $\nu_{y}=2.09$ in steps of 0.02 . The result was a dramatic increase in the rms beam width from $17 \mathrm{~mm}$ at the highest tune value to $22.5 \mathrm{~mm}$ at the lowest tune value. The corresponding experimental losses increased linearly with decreasing tune value up until the last data point, where losses jumped substantially, most likely caused by a violation of the limiting ring aperture.

Finally, beam broadening was measured as a function of painting scheme. A $5 \mu C$ beam was painted vertically to two different sizes: one beam was painted with a $17 \mathrm{~mm}$ 


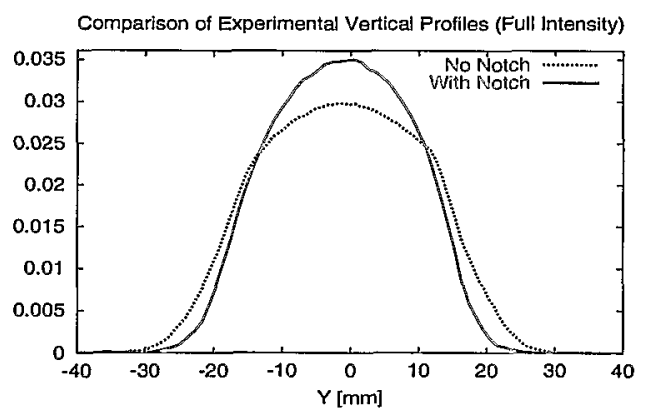

Figure 4: Vertical experimental beam profiles for a $7 \mu C$ beam with and without a longitudinal notch. The red solid line is the beam without the longitudinal notch (bunching factor $\approx .3$ ) and the blue dashed beam is the beam with the longitudinal notch (bunching factor $\approx .45$ ).

maximum injection offset, and the other beam was painted with a $12 \mathrm{~mm}$ maximum injection offset. Profiles recorded at half of the run intensity $(2.5 \mu \mathrm{C})$ show that the smaller painted beam is smaller at the end of accumulation than the larger painted beam. However, with the full $5 \mu C$ of beam current, the small and large painted vertical beam profiles at the end of accumulation are nearly identical (see Figure 5). The smaller painted beam, subject to a much more intense space charge environment, reacts more quickly and more severely to the coherent resonance than does the large painted beam.

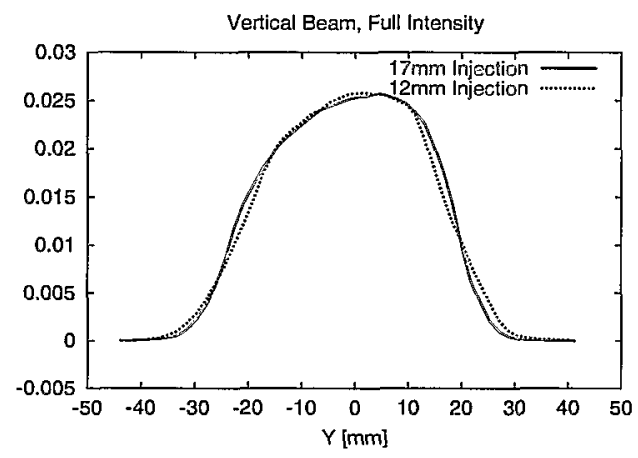

Figure 5: Vertical experimental profiles after accumulation of a $5 \mu C$ beam painted to $17 \mathrm{~mm}$ and a beam painted to $12 \mathrm{~mm}$. The solid red is the large painted beam and the dashed blue is the small painted beam.

PIC simulations of the experiment show that the small painted beam reacts to the resonance very early in the accumulation stage, and displays beam moment oscillations that are about $10 \%$ larger than those of the large painted beam. After this initial stage of growth, the small painted beam oscillates with moment amplitudes just slightly higher than those of the large painted beam, and the emittance of the small beam at the end of accumulation is about $8 \%$ greater than that of the large beam. This behavior is consistent with Sacherer's claims that the emittance of the beam grows in response to the half integer resonance and. weakens the space charge environment. In an accumulation scenario, this is an ongoing process, and a comparison of Sacherer's analytic equations with the PIC simulations shows that the PSR beam is sitting continuously at the very edge of the coherent resonance threshold. The emittance of the beam is growing constantly in excess of pure painting effects in order to compensate for the resonance condition. Eventually the beam size exceeds the limiting ring aperture and losses escalate, ultimately limiting the attainable beam intensity.

\section{CONCLUSIONS}

Strong evidence supports the conclusion that beam broadening at high intensities in the PSR accumulator ring is a result of a coherent half-integer resonance response. PIC simulations, all well-benchmarked with experimental data, show that space-charge-depressed tunes are in excess of the incoherent stopband limit before the onset of broadening, and that the beam envelope executes four oscillations per turn, both strong indicators of a coherent resonance. Experiments performed to explore the resonance show that at high intensity, factors such as longitudinal profile shape, transverse painting scheme, and variation of bare tune all affect the response of the beam to the resonance; at low intensities, before the onset of resonance, no significant changes are observed from the modification of these parameters.

This work is supported by SNS through UT-Battelle, LLC, under contract DE-AC05-00OR22725 for the U.S. DOE, and by Indiana University through a DOE grant, DEFG02-92ER40747. SNS is a partnership of six national laboratories: Argonne, Brookhaven, Jefferson, Lawrence Berkeley, Los Alamos, and Oak Ridge. The Los Alamos National Laboratory is operated by the Univesity of California for the U.S. DOE under contract W-7405-ENG-36.

\section{REFERENCES}

[1] J.A. Holmes, V. Danilov, J. Galambos, A. V. Fedotov, and R. L. Gluckstern, in Proceedings of the Particle Accelerator Conference, Chicago, 2001, p. 3188.

[2] A. V. Fedotov and I. Hofmann, "Half-Integer Resonance Crossing in High-Intensity Rings," PRST-AB 5, (2002) 024202 .

[3] A. Uesugi, S. Machida, and Yoshiharu Mori, "Experimental study of a half-integer resonance with space-charge effects in a synchotron," PRST-AB 5, (2002) 044201.

[4] F. J. Sacherer, "Transverse Space-Charge Effects in Circular Accelerators," PhD Thesis, University of California, Berkeley, (1968).

[5] S.Y. Lee and H. Okamoto, Phy. Rev. Lett. 80, 124201 (1999).

[6] J. Holmes, J. Galambos, D. Olsen, S.Y. Lee, in Workshop on Space Charge Physics in High Intensity Hadron Rings, Shelter Island, NY, 1998, p. 254.

[7] J. Galambos et al., ORBIT User's Manual, SNS/ORNL/AP Technical Note 011, 1999. 